\title{
Continuing Cancer-Directed Therapy in Children with COVID-19 Infection-Adopting Newer Strategies?
}

\author{
Shuvadeep Ganguly ${ }^{1}$. Sameer Bakhshi ${ }^{1}$
}

Received: 28 January 2022 / Accepted: 2 February 2022 / Published online: 25 February 2022

c) Dr. K C Chaudhuri Foundation 2022

During initial days of the ongoing coronavirus 19 (COVID19) pandemic, concerns regarding impact of cancer therapy on severity of COVID-19 in children along with lockdowns imposed by governments led to significant disruption of cancer care across the world. Several institutions adapted their treatment protocol to ensure continuation of care while curtailing unnecessary hospital visits $[1,2]$. Yet, systematic studies of impact of COVID-19 infection and treatment disruptions on cancer care of children are few, especially in resource-constrained settings. In this issue of the Journal, the study by Parambil et al. provides an important snapshot of experience of managing COVID-19 infection among children with cancer along with strategies for treatment adaptations [3].

It is pertinent to note that, although majority of children (94.2\%) had only mild disease, COVID-19-related mortality in this cohort was $4.9 \%$, which is relatively higher than that observed in children in general [3]. This reiterates the need for adopting newer strategies to limit the infection in this vulnerable cohort of children. An important strategy in this aspect is strengthening telemedicine services and using a "shared care" model to provide cancer care without any physical hospital visits [4]. This is feasible especially in children undergoing less intensive treatment like maintenance therapy of acute lymphoblastic leukemia (ALL) or those on relatively simpler protocols as for Hodgkin lymphoma. Utilizing a "shared care" model by involving local pediatric healthcare in delivering cancer care also has the beneficial consequence of disseminating pediatric oncology care across country with reduction of healthcare burden on tertiary care institutions.

Sameer Bakhshi

sambakh@hotmail.com

1 Department of Medical Oncology, Dr. B.R.A. Institute Rotary Cancer Hospital, All India Institute of Medical Sciences, New Delhi 110029, India
On an encouraging note, among children on active treatment, 90.9\% (70/77) could receive their planned treatment protocol with a median delay of $14 \mathrm{~d}$. Further treatment decision in such a scenario should be tailored according to treatment intent. While for patients with curative intent, it is reasonable to continue intensive treatment at earliest opportunity feasible; for palliative intent patients, utilizing alternative options like home-based oral metronomic therapy with similar outcome is more prudent. This also has the added advantage of reduced toxicities, reduced hospital visits, and improved quality of life of children [5].

We observe that the authors [3] have serially monitored for viral clearance by reverse-transcriptase-polymerase chain reaction (RT-PCR) assay in all patients, although the relevance of such practice on subsequent decision on treatment reinitiation is questionable. Indeed, treatments were reinitiated in clinically stable patients $1 \mathrm{wk}$ from RT-PCR positivity irrespective of viral clearance without any adverse effect. Notably, in newly diagnosed patients of ALL having high leucocyte count or aggressive lymphomas, cancer therapy may be initiated even in those having active COVID19; a delay in such an emergent situation is likely to be fatal.

While, immunosuppression due to cancer-directed therapy reflected by lower nadir absolute neutrophil count (ANC) may be responsible for delayed RT-PCR negativity, it is unclear why delayed viral clearance was observed among children with higher baseline ANC; steroid use or type of malignancy did not affect viral clearance [3]. Further studies on immunogenicity following COVID-19 infection among children with cancer, based on type of cancer and antineoplastic therapy, need to be conducted systematically.

\section{Declarations}

Conflict of Interest None. 


\section{References}

1. Pushpam D, Bakhshi S, Agarwala S. Chemotherapy adaptations in a referral tertiary care center in India for ongoing therapy of pediatric patients with solid tumors during COVID19 pandemic and lockdown. Pediatr Blood Cancer. 2020;67:e28428.

2. Garg V, Bakhshi S, Gupta G, Pushpam D. Managing pediatric cancer patients in COVID19 pandemic. Indian J Pediatr. 2020;87:558.

3. Parambil BC, Moulik NR, Dhamne C, et al. COVID-19 in children with cancer and continuation of cancer-directed therapy during the infection. Indian J Pediatr. 2021. https://doi.org/10.1007/ s12098-021-03894-3.

4. Ganguly S, Bakhshi S. Teleconsultations and shared care in pediatric oncology during COVID-19. Indian J Pediatr. 2021;88:1-2.

5. Bahl A, Bakhshi S. Metronomic chemotherapy in progressive pediatric malignancies: old drugs in new package. Indian J Pediatr. 2012;79:1617-22.

Publisher's Note Springer Nature remains neutral with regard to jurisdictional claims in published maps and institutional affiliations. 